\title{
Analyse Comparative De La Rentabilite Economique De L'exportation De L'ananas Frais Et De Son Jus Au Benin
}

\begin{abstract}
Chabi Felix Biaou
Faculté des Sciences Economiques et de Gestion (FASEG)/UAC Cotonou Docteur en économie rurale, Enseignant à la FASEG /UAC, MaitreAssistant, Benin

Doi:10.19044/esj.2018.v14n31p399 URL:http://dx.doi.org/10.19044/esj.2018.v14n31p399

Abstract

In recent years, the rapid increase in pineapple production has been accompanied by an increase in its exports and that of its juice to various subregions. To assess the profitability of these exchanges, surveys were conducted among producers, processors, and exporters. The Cost-Financial and Economic Benefit ratios, nominal and effective protection coefficients, and the ratio of comparative advantage were used in this study. The results show that the export of fresh pineapple to hinterland countries is, on the one hand, more profitable than that of pineapple juice because of the proliferation of sugar-sweetened beverages in the sub-region. On the other hand, it is more profitable than its export to Europe.
\end{abstract}

Keywords: Fresh pineapple, financial and economic cost-benefit ratios, domestic resource costs, tradable and non-tradable factors

\section{Résumé}

L'accroissement fulgurant de la production d'ananas ces dernières années s'est accompagné de la hausse de son exportation et de celle de son jus, vers la sous-région. Pour apprécier la rentabilité de ces échanges, des enquêtes ont été réalisées auprès des producteurs, des transformateurs et des exportateurs. Les ratios Coûts- Bénéfices financiers et économiques, les coefficients de protection nominale et effective et le ratio de l'avantage comparatif ont été utilisés. Les résultats montrent que l'exportation de l'ananas frais vers les pays de l'hinterland est, d'une part, plus rentable que celle du jus d'ananas à cause de la prolifération des boissons sucrées dans la sous-région et d'autre part plus rentable que son exportation vers l'Europe. 
Mots-clés : Ananas frais, ratios coûts- bénéfices financiers et économiques, coûts en ressources intérieures, facteurs échangeables et non échangeables

\section{INTRODUCTION}

Un système de commercialisation efficace ne sert pas seulement de lien entre le producteur et le consommateur, mais il contribue activement et positivement au développement en stimulant la consommation. L'efficacité économique du système commercial permet de mesurer la performance avec laquelle les fonctions commerciales s'accomplissent afin de prendre des mesures correctives. Si le système de prix fonctionne convenablement, il incite à satisfaire de plus en plus la demande des consommateurs par rapport au type, à la qualité et au délai de livraison (Obi et al., 2011). Ainsi, la production s'adapte à la demande en réagissant « aux signaux de prix » émis par le système de commercialisation. Harris (1982) avance que la commercialisation des produits agricoles génère trois utilités principales: l'utilité du lieu, celle du temps et celle de la forme. En effet, elle constitue un moyen de parvenir aux buts fondamentaux de la société qui sont la croissance économique, la répartition plus équitable des revenus, le bien-être nutritionnel et la sécurité alimentaire (Timmer et al., 1986).

Un système de commercialisation efficace doit être orienté vers les besoins des consommateurs et générer un profit pour tout acteur impliqué, à savoir le producteur, le transporteur, le commerçant, le transformateur, etc. (Timmer, 1986). Cependant, à cause des dysfonctionnements du système commercial, les commerçants sont souvent qualifiés d'intermédiaires inutiles, de spéculateurs, faisant penser que les activités commerciales sont inutiles et contraires aux intérêts de la société. Ce dysfonctionnement s'opère surtout dans les pays en voie de développement où le manque d'infrastructures routières, de moyens de transport et de communication et des normes font défaut (Banque Mondiale, 2012). Selon Stiglitz et Greenwald (1989), la décadence de l'économie des pays les moins avancés n'est pas uniquement liée au manque du capital physique ou humain, mais aussi à l'inefficacité du fonctionnement de leur marché.

Le commerce extérieur étant un déterminant important de la croissance économique à long terme, le système commercial permet aussi de montrer qu'un pays ou une région est compétitif ou non dans la production et l'exportation d'un bien. Ainsi, les politiques économiques privilégiant la croissance des exportations, les investissements et la libéralisation commerciale sont au cœur des stratégies recommandées aux pays en développement (Banque Mondiale, 2008). Les gains tirés de la libéralisation commerciale viennent d'une part des avantages comparatifs qui peuvent être sous forme de dotations en ressources naturelles (modèle Hecksher-Ohlin) ou de différences technologiques (modèle ricardien) et d'autre part elle 
accroît le revenu par tête et la croissance à travers l'économie d'échelle et la diffusion technologique entre les pays.

La compétitivité d'un pays est fondée sur la notion de l'avantage comparatif. Pour ce faire, divers indicateurs sont classés en indicateurs fondés sur les échanges et indicateurs fondés sur la gestion stratégique (Latruffe, 2010). Les indicateurs fondés sur les échanges portent sur le taux de change réel ( Real Effective Exchange Rate (REER), la parité du pouvoir d'achat (PPP- Purchasing Power Parity), l'avantage comparatif révélé (RCARevealed Comparative Advantage) et ses dérivés tels que l'avantage relatif à l'exportation (REA- Revealed Exportation Advantage), l'avantage relatif à l'importation (RIA-Revealed Importation Advantage), l'avantage commercial relatif (RCA- Revealed Commercial Advantage), l'indice des exportations nettes (NEI- Net Exports Index), etc. et les indicateurs fondés sur la gestion stratégique regroupent les indicateurs de coûts, les indicateurs de rentabilité et les indicateurs de productivité. Les indicateurs de mesure des coûts portent sur le ratio coût en ressources intérieures (CIR-Cost of Internal Ressources), le ratio coûts avantages sociaux (SBC- Social Benefits Cost) et les coûts de production.

Du point de vue empirique, Mulder et al. (2004) examinant la compétitivité de l'agriculture et du secteur agroalimentaire dans les pays du Mercosur et de l'UE au cours de la période 1991-99 ont montré, à partir des REER, que les pays du Mercosur enregistrent une baisse de la compétitivité depuis 1998 (à l'exception du Paraguay pour lequel elle est demeurée stable). Gorton et al. (2000) utilisant le ratio CRI pour les principaux produits agricoles de la Bulgarie et la République Tchèque au cours de la période 1994-96, montrent que le blé et l'orge sont plus compétitifs pour les deux pays, à la fois à l'échelle mondiale et de l'UE. Gorton et Davidova (2001) passent en revue plusieurs études analysant la compétitivité internationale de l'agriculture des pays du PECO (Bulgarie, République Tchèque, Hongrie, Pologne, Roumanie, Slovaquie, Slovénie) entre 1992 et 1998, sur la base du ratio CRI et des données au niveau de l'exploitation. Ils constatent que les produits végétaux sont plus compétitifs au plan international que les produits animaux et que, dans le domaine des grandes cultures, ce sont le blé et le tournesol qui sont les plus compétitifs. Après l'examen des études utilisant le ratio CRI pour les mêmes pays du PECO entre 1989 et 1998, Bojnec (2003) conclut lui aussi que la production animale est moins compétitive au plan international que la production végétale.

La perpétuation de la production de l'ananas, essentiellement cultivé dans le département de l'Atlantique au sud du pays, dépend de l'efficacité de sa commercialisation. Or, selon Biaou et al. (2016) près de $80 \%$ de l'ananas béninois et plus de $80 \%$ du jus extrait sont exportés vers la sous-région. 
L'objectif de cet article est de comparer la rentabilité économique de l'exportation de l'ananas frais et de son jus vers la sous-région.

\section{APPROCHES MÉTHODOLOGIQUES}

\subsection{Méthodes d'analyse}

Comme les prix du marché ne reflètent pas la vraie valeur économique des biens à cause des distorsions dans leur fonctionnement, la Matrice d'Analyse des Politiques (MAP) utilise les prix économiques pour les corriger, pour faire apparaître les écarts entre les comptes économiques ainsi reconstitués et les comptes financiers des agents et pour analyser les impacts des politiques gouvernementales sur les prix et sur la compétitivité des filières (Pearson \& Monke, 1989).

Structurellement, la MAP est composée de deux types de budgets : un budget évalué au prix du marché ou prix financier (budget financier) et l'autre aux coûts d'opportunité social ou prix économique (budget économique) où les valeurs (prix, coûts, profits) sont celles qu'on observerait dans une situation de libre-échange ou d'absence de distorsions (Tableau 1). Pour ce faire, tous les facteurs du système de production sont répartis en facteurs échangeables et non échangeables.

Tableau 1. Structure de la matrice d'analyse des politiques

\begin{tabular}{|c|c|c|c|c|}
\hline \multirow[t]{2}{*}{ Indicateurs } & \multirow[t]{2}{*}{ Revenus } & \multicolumn{2}{|c|}{ Coûts des facteurs } & \multirow[t]{2}{*}{ Profit } \\
\hline & & échangeables & non échangeables & \\
\hline Budget financier (prix du marché) & $\mathrm{A}_{\mathrm{ij}}$ & $\mathrm{B}_{\mathrm{ij}}$ & $\mathrm{C}_{\mathrm{ij}}$ & $\mathrm{D}_{\mathrm{ij}}$ \\
\hline Budget économique (prix de référence) & $E_{i j}$ & $\mathrm{~F}_{\mathrm{ij}}$ & $\mathrm{G}_{\mathrm{ij}}$ & $\mathrm{H}_{\mathrm{ij}}$ \\
\hline Divergences ou convergences & $\mathrm{I}_{\mathrm{ij}}$ & $\mathrm{J}_{\mathrm{ij}}$ & $\mathrm{K}_{\mathrm{ij}}$ & $\mathrm{L}_{\mathrm{ij}}$ \\
\hline
\end{tabular}

Source : Pearson \& Monke (1989).

Les $\mathrm{i}$ traduisent les produits et les $\mathrm{j}$ les destinations des produits. Les paramètres des deux premières du Tableau 1 sont :

$\mathrm{A}_{\mathrm{ij},}$, le revenu brut financier de la vente du bien i ;

$\mathrm{B}_{\mathrm{ij}}$, les coûts financiers des facteurs échangeables pour la production du bien i ;

$\mathrm{C}_{\mathrm{ij}}$, les coûts financiers des facteurs non échangeables pour la production du bien i ;

$\mathrm{D}_{\mathrm{ij}}$, le profit financier de la vente du bien i ;

$\mathrm{E}_{\mathrm{ij}}$, le revenu économique de la vente du bien i ;

$\mathrm{F}_{\mathrm{ij}}$, les coûts économiques des facteurs échangeables pour la production du bien i ;

$\mathrm{G}_{\mathrm{ij}}$, les coûts économiques des facteurs non échangeables pour la production du bien i ;

$\mathrm{H}_{\mathrm{ij}}$, le profit économique issu de la production du bien $\mathrm{i}$. 
Les divergences ou convergences qui sont les différences par colonne des éléments de la première ligne et de ceux de la deuxième ligne ne sont pas utilisées dans cet article. Les indicateurs utilisés pour mesurer la rentabilité des échanges de l'ananas et de son jus sont les ratios Coûts Bénéfices financiers (RCBF), économiques (RCBE), le coût en ressources intérieures (CRI) et les coefficients de protection nominale des produits (CPNP) et effective (CPE).

Le $\mathrm{RCBF}_{\mathrm{ij}}$ est une mesure directe de la motivation des producteurs à produire le bien qui reflète la compétitivité ou l'efficacité du système de production. Il a pour expression :

$\operatorname{RCBF}_{\mathrm{ij}}=\frac{\left(\mathrm{B}_{\mathrm{ij}}+\mathrm{C}_{\mathrm{ij}}\right)}{\mathrm{A}_{\mathrm{ij}}}$

Le $\mathrm{RCBE}_{\mathrm{ij}}$ mesure l'importance des coûts économiques. Il présente la rentabilité économique sociale de l'activité et se calcule par :

$\operatorname{RCBE}_{\mathrm{ij}}=\frac{\left(\mathrm{F}_{\mathrm{ij}}+\mathrm{G}_{\mathrm{ij}}\right)}{\mathrm{E}_{\mathrm{ij}}}$.

L'avantage comparatif est apprécié par le ratio du coût en ressources intérieures (CRI) qui mesure l'efficacité de la meilleure utilisation des ressources locales ; c'est-à-dire, le coût d'opportunité de la production d'une unité du produit en employant les ressources locales. Il est défini comme la valeur de référence des facteurs non échangeables utilisés par unité des facteurs échangeables.

$$
C R I_{i j}=\frac{G_{i j}}{E_{i j}-F_{i j}}
$$

Ainsi lorsque le $\mathrm{CRI}_{\mathrm{ij}}$ est compris entre 0 et un, le pays a un avantage comparatif dans la production du bien sur la base de la technologie considérée. Autrement dit, l'activité de production est économiquement efficace et moins coûteuse en ressources intérieures. Le système étudié utilise moins de ressources internes qu'il ne génère de valeur ajoutée. Un tel système permet de gagner des devises. Mais lorsque $\mathrm{CRI}_{\mathrm{ij}}$ est supérieur à l'unité, il indique au contraire que le système utilise plus de ressources intérieures (travail et capital) qu'il ne génère de valeur ajoutée. Lorsque $\mathrm{CRI}_{\mathrm{ij} e s t}$ égal à un, il traduit un cas d'indifférence ; c'est-à-dire, que le producteur ne réalise ni bénéfice ni perte en produisant localement ou en important le bien.

Le coefficient de protection nominale pour les produits et (CPNP) mesure le rapport de la valeur des produits au prix du marché sur leur valeur au prix de référence. Lorsque le CPNP est supérieur à l'unité, il indique que 
les produits sont subventionnés et lorsqu'il est inférieur à l'unité, les produits sont taxés.

$\operatorname{CPNP}_{\mathrm{ij}}=\frac{\mathrm{A}_{\mathrm{ij}}}{\mathrm{E}_{\mathrm{ij}}}$

Si q désigne le CPNP, le producteur ne perçoit que $A=q$ * $E$. Le système est donc taxé de $t=(1-q) \star 100$

Le coefficient de protection effective (CPE) est une mesure agrégée $\mathrm{du}$ taux de protection du système productif qui prend en compte simultanément les effets des distorsions sur le marché des produits et sur celui des intrants échangeables.

$\mathrm{CPE}_{\mathrm{ij}}=\frac{\mathrm{A}_{\mathrm{ij}}-\mathrm{B}_{\mathrm{ij}}}{\mathrm{E}_{\mathrm{ij}}-\mathrm{F}_{\mathrm{ij}}}$

Un CPE inférieur à 1 signifie que la combinaison des transferts sur les produits d'une part, et sur les consommations intermédiaires (biens échangeables) d'autre part, résulte en ce qui suit :

- $\quad$ une distribution effective des revenus inférieure à ce qu'elle devait être en cas d'application, toutes choses égales par ailleurs, des prix internationaux ;

une valeur ajoutée distribuée aux agents moindre de ce qu'elle représente économiquement pour la collectivité (Fabre, 1994).

Plus ces coefficients sont faibles, mieux vaut l'avantage dont dispose le pays à produire le bien ou le service.

\subsection{Méthodes de collecte des données}

\subsubsection{Zone de recherche}

Cette recherche est conduite dans le département de l'Atlantique qui produit plus de $98 \%$ de l'ananas au Bénin. Il est une zone de forte densité démographique proche des grands centres urbains du pays comme Cotonou, Porto-Novo, Abomey-Calavi, etc. Sa population estimée à plus de 800000 habitants (INSAE, 2013) est répartie dans huit (08) communes dont cinq sont les principales productrices d'ananas.

\subsection{2. Échantillonnage et technique et outils de collecte des données}

Les données primaires utilisées dans le cadre de cet article proviennent des enquêtes auprès des producteurs, des commerçants et des transformateurs.

Dix (10) producteurs sont sélectionnés de façon aléatoire dans les quarante (40) villages retenus et répartis dans les cinq communes productrices d'ananas du département. La commune et le village sont les deux strates considérées dans ce travail. Les villages retenus ont été répartis 
entre les communes proportionnellement au nombre de villages producteurs d'ananas dans le département et les villages à enquêter dans chaque commune sont déterminés en fonction du poids de la superficie de l'ananas du village dans celle de la commune. Sur les 400 producteurs ciblés, 365 ont répondu à notre interview.

De même, à partir de la liste des producteurs de jus obtenue auprès de l'Association Nationale des transformateurs d'Ananas au Bénin (ANATRAB), 45 producteurs ont été sélectionnés de façon aléatoire. Nous avons eu des discussions avec le bureau de l'ANATRAB sur l'exportation du jus dans la sous-région. Au niveau des commerçants où une telle liste n'est pas disponible, nous les dénombrons dans chacun des marchés les jours de leur animation et nous les abordons et nous prenons rendez-vous à domicile avec certains ou ailleurs hors du marché. Pour les détaillants ambulants, nous profitons de nos achats pour discuter et avoir rendez-vous avec eux afin de les interviewer par notre questionnaire. De cette manière nous avons pu questionner 110 commerçants dont 5 exportateurs d'ananas frais.

\subsubsection{Données collectées}

Les données sont collectées auprès de ces différents acteurs par entretien individuel à l'aide d'un questionnaire. Au niveau des producteurs d'ananas frais, elles ont porté sur les superficies et quantités récoltées et vendues, les quantités des intrants utilisés par ha (engrais, carbure, rejets), le sexe du producteur, son âge, la main-d'œuvre utilisée par hectare et par opération culturale, les coûts de location de la terre et de la main-d'œuvre, les équipements et matériels utilisés, leurs coûts et durées de vie, le nombre de fois que ces matériels sont utilisés dans les champs d'ananas, etc. Celles des commerçants ont porté sur les modes d'achat (préfinancement, à crédit ou au comptant), les destinations du produit, les prix d'achat et de vente, les coûts de récolte, les charges commerciales (transport, conditionnement, manutention, et faux frais aussi bien à la douane qu'au cours du trajet), les chargements et déchargements, les unités d'achat et de vente, le niveau d'instruction, l'âge, les activités secondaires et les difficultés rencontrées. $\mathrm{Au}$ niveau des transformateurs, les données ont porté sur les quantités d'ananas transformé, les quantités de jus commercialisé, les destinations, les coûts de transport, les frais de route, de manutention et les faux frais, les charges d'emballage, etc.

Les données secondaires sont recueillies auprès des institutions comme la Chambre d'Agriculture, la Chambre de Commerce et d'Industrie du Bénin (CCIB), l'INSAE, la douane, etc. et ont porté sur les pratiques en matière d'exportation et d'importation des produits agricoles et surtout l'ananas et ses dérivés, les taxes et impôts, les subventions et autres charges à l'exportation. 
Par rapport aux données mercuriales, nous avons utilisé le prix moyen de 2015 fourni par le journal FRUITOP pour la France, principale importatrice de l'ananas béninois. Les données mercuriales des fruits étant non disponibles dans les pays de la sous-région, nous avons utilisé les données de l'ABC/SNV (2016) qui divergent très peu de celles que nous avons eues par échanges téléphoniques mensuellement avec les amis et parents des capitales de ces pays.

\section{RÉSULTATS ET DISCUSSIONS}

\subsection{Principales zones d'exportation de l'ananas et de son jus}

La production de l'ananas au Bénin, concentrée dans la partie méridionale du pays, excède les trois cent mille tonnes depuis 2013. Le volume exporté vers l'Europe représente moins de 5\% de cette production (Biaou et al., 2016). Les tracasseries policières ne permettent pas de ravitailler régulièrement toutes les régions du pays. Ainsi, la zone de sa production est saturée alors que des régions Nord-Ouest et Sud-Ouest du pays le reçoivent accidentellement (Adegbola \& Arouna, 2008). Bien que densément peuplée, cette partie méridionale du pays se trouve nettement incapable de consommer le reste de cette production qui est alors déversée dans la sous-région. Ainsi, près de $80 \%$ de l'ananas béninois est exporté vers le Nigeria et les pays limitrophes.

Par ailleurs, plus de 6\% de cette production d'ananas est transformé sur place (Biaou et al., 2016). Le jus en est le principal dérivé qui se vend également dans la sous-région et qui est commercialisé surtout dans les pays de l'hinterland (Niger Burkina) et au Sénégal (ABC/SNV, 2016). La plupart des transformateurs de l'ananas en jus affirment exporter plus de $80 \%$ de leurs productions dans la sous-région.

\subsection{Ratios Coûts Bénéfices de l'exportation de l'ananas et du jus}

Les ratios Coûts-Bénéfices financiers ( $\mathrm{RCBF}$ ) et économiques (RCBE) sont partout inférieurs à l'unité, aussi bien pour l'ananas frais que pour le jus. Mais les ratios financiers sont partout supérieurs aux ratios économiques (Tableau 2).

L'analyse des ratios Coûts-Bénéfices financiers (RCBF) de l'exportation de l'ananas frais montre qu'ils varient d'un producteur à l'autre, suivant le genre, le niveau d'instruction, le milieu de vie et la variété cultivée. Ces ratios moyens ne sont statistiquement différents que suivant la variété cultivée (au seuil de 1\%), où ils sont respectivement de 0,5436 pour la Cayenne lisse et de 0,8461 pour le pain de sucre. Mais les RCBF moyens du jus ne sont pas statistiquement différents quel que soit le sous-groupe considéré. 
Les ratios Coûts-Bénéfices économiques (RCBE) de l'exportation de l'ananas frais varient également d'un producteur à l'autre mais aussi suivant les régions d'exportation. Leurs moyennes ne sont statistiquement différentes que par rapport à la variété cultivée au seuil de $1 \%$ partout.

Globalement ces ratios moyens sont plus élevés avec l'Europe $(0,6222)$ qu'avec les pays de l'hinterland $(0,1243)$ et le Nigeria $(0,1133)$. Ainsi, l'exportation de l'ananas frais vers l'Europe coûte économiquement 5,01 fois plus chère qu'avec l'hinterland et 5,49 fois plus chère qu'avec le Nigeria. On comprend pourquoi les exportations informelles sous-régionales dominent l'exportation formelle vers l'Europe bien que la qualité de cet ananas y soit bien appréciée (Arouna \& Afomassè, 2005 ; Arinloyé, 2013). 
Tableau 2. Ratios Coûts bénéfices financiers (RCBF) et économiques (RCBE) moyens de l'exportation de l'ananas frais et de son jus suivant les régions d'exportation de l'ananas et des paramètres liés aux producteurs

\begin{tabular}{|c|c|c|c|c|c|c|c|c|c|}
\hline \multirow{3}{*}{$\begin{array}{l}\text { Paramètr } \\
\text { es }\end{array}$} & \multirow{3}{*}{ Modalités } & \multirow{2}{*}{\multicolumn{2}{|c|}{$\begin{array}{l}\text { Ratios Coûts- Bénéfices } \\
\text { Financiers (RCBF) }\end{array}$}} & \multicolumn{6}{|c|}{ Ratios Coûts -Bénéfices Économiques (RCBE) } \\
\hline & & & & \multicolumn{3}{|c|}{ Ananas frais } & \multicolumn{3}{|c|}{ Jus d'ananas } \\
\hline & & Ananas & Jus & Hinterland & Nigeria & Europe & Hinterland & Nigeria & Sénégal \\
\hline \multirow[t]{3}{*}{ Genre } & Homme & $0,811(0,459)$ & $0,721(0,166)$ & $0,124(0,024)$ & $0,114(0,034)$ & $0,622(0,016)$ & $0,562(0,146)$ & $0,319(0,097)$ & $0,304(0,076)$ \\
\hline & Femme & $0,630(0,322)$ & $0,877(0,392)$ & $0,120(0,022)$ & $0,107(0,031)$ & $0,618(0,012)$ & $0,622(0,257)$ & $0,381(0,245)$ & $0,358(0,191)$ \\
\hline & F de Fischer & 2,45 & 2,409 & 0,554 & 0,551 & 1,02 & 0,775 & 0,983 & 1,221 \\
\hline \multirow{6}{*}{$\begin{array}{c}\text { Niveau } \\
\text { d'instruct } \\
\text { ion }\end{array}$} & Aucun & $0,808(0,394)$ & & $0,126(0,023)$ & $0,115(0,033)$ & $0,623(0,015)$ & - & - & 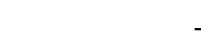 \\
\hline & Primaire & $0,831(0,506)$ & $0,676(0,133)$ & $0,126(0,029)$ & $0,116(0,040)$ & $0,623(0,018)$ & $0,550(0,158)$ & $0,271(0,124)$ & $0,267(0,096)$ \\
\hline & $\begin{array}{l}\text { Secondaire } 1^{\mathrm{er}} \\
\text { cycle }\end{array}$ & $0,803(0,570)$ & $0,606(0,103)$ & $0,121(0,022)$ & $0,109(0,031)$ & $0,621(0,014)$ & $0,440(0,101)$ & $0,233(0,076)$ & $0,238(0,059)$ \\
\hline & $\begin{array}{l}\text { Secondaire } 2^{\text {nd }} \\
\text { cycle }\end{array}$ & $0,727(0,323)$ & $0,840(0,251)$ & $0,116(0,017)$ & $0,102(0,024)$ & $0,616(0,011)$ & $0,622(0,166)$ & $0,372(0,133)$ & $0,345(0,104)$ \\
\hline & Supérieur & $0,595(0,230)$ & $0,882(0,429)$ & $0,114(0,014)$ & $0,099(0,020)$ & $0,615(0,010)$ & $0,628(0,286)$ & $0,391(0,272)$ & $0,368(0,211)$ \\
\hline & F de Fischer & 0,667 & 0,822 & 1,589 & 1,591 & 1,575 & 0,684 & 0,684 & 0,759 \\
\hline \multirow[t]{2}{*}{ Variétés } & Cayenne lisse & $0,544(0,331)$ & & $0,113(0,018)$ & $0,097(0,025)$ & $0,616(0,013)$ & & & \\
\hline & Pain de sucre & $0,846(0,459)$ & & $0,126(0,025)$ & $0,116(0,035)$ & $0,623(0,016)$ & & & \\
\hline \multirow{4}{*}{$\begin{array}{l}\text { Technolo } \\
\text { gie }\end{array}$} & F de Fischer & $22,012^{* * * *}$ & & $14,92 * * *$ & $14,93 * * *$ & $12,82 * * *$ & & & \\
\hline & Non motorisée & & $0,797(0,327)$ & & & & $0,560(0,159)$ & $0,328(0,140)$ & $0,311(0,110)$ \\
\hline & Motorisée & & $0,832(0,340)$ & & & & $0,626(0,255)$ & $0,378(0,237)$ & $0,355(0,184)$ \\
\hline & $\mathrm{F}$ de Fischer & & 0,113 & & & & 0,938 & 0,646 & 0,852 \\
\hline \multicolumn{2}{|c|}{ Ensemble } & $0,803(0,455)$ & $0,818(0,332)$ & $0,124(0,024)$ & $0,113(0,034)$ & $0,622(0,016)$ & $0,600(0,222)$ & $0,358(0,203)$ & $0,337(0,159)$ \\
\hline
\end{tabular}

Source : Données de l'enquête, 2013 et 2015. ( ) Ecart type ;** significatif à $1 \%$. 


\subsection{Avantages comparatifs (CRI) de l'exportation de l'ananas}

\section{frais et du jus.}

Les coefficients d'utilisation des ressources intérieures sont partout inférieurs à l'unité, faisant preuve de que le Bénin a un avantage comparatif par rapport à ces pays dans la production de l'ananas et du jus de ce fruit. Ces coûts en ressources intérieures variables d'un producteur à l'autre sont très variables d'une région d'exportation à l'autre. L'examen de ce coefficient suivant les zones d'exportation de l'ananas montre qu'il est très élevé pour l'Europe et très faible pour les pays de l'hinterland. L'exportation de l'ananas frais vers l'Europe coûte en moyenne 5,73 fois plus en ressources intérieures que son exportation vers les pays de l'hinterland et 4,19 plus que celle vers le Nigeria (Tableau 3). Ces fortes pertes en ressources expliquent pourquoi l'ananas béninois est plus exporté vers la sous-région que vers l'Europe. En effet, ces forts CRI pour l'Europe sont essentiellement dus aux frais de manutention et de fret qui constituent plus de $57 \%$ des frais d'exportation de l'ananas.

L'examen de ces coefficients suivant les paramètres liés aux producteurs montre qu'ils sont statistiquement différents suivant les variétés cultivées au seuil de 1\% partout (Tableau 3). Ces ratios confirment la rentabilité de l'exportation sous-régionale du jus d'ananas. Globalement, ils ont pour moyennes respectives de 0,$1023 ; 0,1288$ et 0,3982 pour le Sénégal, le Nigeria et l'hinterland. Pourtant, l'exportation de l'ananas frais est plus rentable vers les pays de l'hinterland que celle du jus qui est plus rentable en l'exportant vers le Sénégal et le Nigeria.Mais les écarts-types du CRI du jus montrent que ce ratio est très variable d'un transformateur à l'autre, prouvant de fortes variabilités des rendements d'extraction du jus. Cette dernière dépend de plusieurs facteurs dont l'état juteux ou non des fruits, la maitrise des techniques d'extraction, la période de production, etc. L'amélioration de ce rendement d'extraction du jus demande l'utilisation des équipements performants et adaptés et la production des variétés d'ananas à haut rendement en jus. 
Tableau 3. Avantages comparatifs (CRI) moyens de l'exportation de l'ananas frais et de son jus vers les pays de l'hinterland, le Nigeria, le Sénégal et l'Europe

\begin{tabular}{|c|c|c|c|c|c|c|c|}
\hline \multirow[t]{2}{*}{ Paramètres } & \multirow[t]{2}{*}{ Modalités } & \multicolumn{3}{|c|}{ Ananas frais } & \multicolumn{3}{|c|}{ Jus d'ananas } \\
\hline & & Hinterland & Nigeria & Europe & Hinterland & Nigeria & Sénégal \\
\hline \multirow[t]{3}{*}{ Genre } & Homme & $\begin{array}{r}0,044 \\
(0,026)\end{array}$ & $\begin{array}{r}0,061 \\
(0,036)\end{array}$ & $\begin{array}{r}0,253 \\
(0,028)\end{array}$ & $\begin{array}{r}0,390 \\
(0,147)\end{array}$ & $\begin{array}{r}0,101 \\
(0,069)\end{array}$ & $\begin{array}{r}0,079 \\
(0,055)\end{array}$ \\
\hline & Femme & $0,040(0,024$ & $\begin{array}{r}0,054 \\
(0,032)\end{array}$ & $\begin{array}{r}0,247 \\
(0,024)\end{array}$ & $\begin{array}{r}0,403 \\
(0,225)\end{array}$ & $\begin{array}{r}0,146 \\
(0,122)\end{array}$ & $\begin{array}{r}0,116 \\
(0,100)\end{array}$ \\
\hline & F de Fischer & 0,53 & 0,531 & 0,678 & 0,049 & 1,934 & 1,986 \\
\hline \multirow[t]{6}{*}{$\begin{array}{c}\text { Niveau } \\
\text { d'instruction }\end{array}$} & Aucun & $\begin{array}{r}0,046 \\
(0,025) \\
\end{array}$ & $\begin{array}{r}0,063 \\
(0,035) \\
\end{array}$ & $\begin{array}{r}0,254 \\
(0,027) \\
\end{array}$ & & & \\
\hline & Primaire & $\begin{array}{r}0,046 \\
(0,031)\end{array}$ & $\begin{array}{r}0,064 \\
(0,042)\end{array}$ & $\begin{array}{r}0,255 \\
(0,033)\end{array}$ & $\begin{array}{r}0,421 \\
(0,191)\end{array}$ & $\begin{array}{r}0,126 \\
(0,128)\end{array}$ & $\begin{array}{r}0,100 \\
(0,102)\end{array}$ \\
\hline & secondaire $1^{\text {er }}$ cycle & $\begin{array}{r}0,041 \\
(0,024)\end{array}$ & $\begin{array}{r}0,056 \\
(0,032)\end{array}$ & $\begin{array}{r}0,250 \\
(0,025)\end{array}$ & $\begin{array}{r}0,276 \\
(0,067)\end{array}$ & $\begin{array}{r}0,053 \\
(0,025)\end{array}$ & $\begin{array}{r}0,042 \\
(0,020)\end{array}$ \\
\hline & $\begin{array}{l}\text { Secondaire } 2^{\text {nd }} \\
\text { cycle }\end{array}$ & $\begin{array}{r}0,035 \\
(0,019)\end{array}$ & $\begin{array}{r}0,049 \\
(0,025)\end{array}$ & $\begin{array}{r}0,243 \\
(0,020)\end{array}$ & $\begin{array}{r}0,453 \\
(0,177)\end{array}$ & $\begin{array}{r}0,138 \\
(0,105)\end{array}$ & $\begin{array}{r}0,107 \\
(0,080)\end{array}$ \\
\hline & Supérieur & $\begin{array}{r}0,033 \\
(0,015)\end{array}$ & $\begin{array}{r}0,045 \\
(0,021)\end{array}$ & $\begin{array}{r}0,240 \\
(0,017)\end{array}$ & $\begin{array}{r}0,375 \\
(0,237)\end{array}$ & $\begin{array}{r}0,132 \\
(0,122)\end{array}$ & $\begin{array}{r}0,107 \\
(0,103)\end{array}$ \\
\hline & F de Fischer & 1,594 & 1,594 & 1,59 & 0,752 & 0,608 & 0,577 \\
\hline \multirow[t]{3}{*}{ Variétés } & Cayenne lisse & $\begin{array}{r}0,032 \\
(0,019)\end{array}$ & $\begin{array}{r}0,044 \\
(0,026)\end{array}$ & $\begin{array}{r}0,240 \\
(0,021)\end{array}$ & & & \\
\hline & Pain de sucre & $\begin{array}{r}0,046 \\
(0,027)\end{array}$ & $\begin{array}{r}0,063 \\
(0,036)\end{array}$ & $\begin{array}{r}0,255 \\
(0,028)\end{array}$ & & & \\
\hline & F de Fischer & $15,073^{* * *}$ & $15,056^{* * *}$ & $14,239 * * *$ & & & \\
\hline \multirow[t]{3}{*}{$\begin{array}{l}\text { Technologie } \\
\text { utilisée }\end{array}$} & Non motorisée & & & & $\begin{array}{r}0,392 \\
(0,167) \\
\end{array}$ & $\begin{array}{r}0,113 \\
(0,103) \\
\end{array}$ & $\begin{array}{r}0,086 \\
(0,074) \\
\end{array}$ \\
\hline & Motorisée & & & & $\begin{array}{r}0,403 \\
(0,218)\end{array}$ & $\begin{array}{r}0,139 \\
(0,110)\end{array}$ & $\begin{array}{r}0,113 \\
(0,094)\end{array}$ \\
\hline & F de Fischer & & & & 0,032 & 0,643 & 1,033 \\
\hline Ensemble & & $\begin{array}{l}0,044 \\
(0,026)\end{array}$ & $\begin{array}{r}0,061 \\
(0,036)\end{array}$ & $\begin{array}{r}0,253 \\
(0,028)\end{array}$ & $\begin{array}{r}0,398 \\
(0,197)\end{array}$ & $\begin{array}{r}0,129 \\
(0,107)\end{array}$ & $\begin{array}{r}0,102 \\
(0,087)\end{array}$ \\
\hline
\end{tabular}

Source : Données de l'enquête, 2013.

( ) Ecart type $* * *$ significatif à $1 \%$.

\subsection{Coefficients de protection nominale de l'ananas frais et du jus}

Le coefficient de protection nominale des produits (CPNP) qui n'est autre que le quotient des chiffres d'affaires financiers et économiques, montre que les producteurs de l'ananas et du jus sont taxés. Ces coefficients montrent que les producteurs d'ananas ne perçoivent que 6,57\%, 9,38\% et $3,73 \%$ des chiffres d'affaires économiques en l'exportant respectivement vers les pays de l'hinterland, le Nigeria et l'Europe alors que les producteurs de jus perçoivent $68,4 \%$; $63,53 \%$ et $49,8 \%$ en l'exportant respectivement vers les pays de l'hinterland, le Nigeria et le Sénégal. Les producteurs d'ananas sont alors plus taxés en exportant vers les pays de l'hinterland et le Nigeria à cause des différents intermédiaires qui gonflent les circuits d'exportation de l'ananas frais. Ainsi, en exportant l'ananas frais vers les pays de l'hinterland par exemple, on peut dire que 93,59\% des chiffres d'affaires économiques échappent aux producteurs et sont transférés vers les 
autres agents par les distorsions du marché et les multiples intermédiaires (Tableau 4).

Par rapport à l'exportation vers l'Europe, ces coefficients expriment les coûts excessifs de manutention, de transit et surtout du fret et d'autres services qui constituent plus de $57 \%$ des coûts de revient de cette exportation. Le problème du coût du fret ne pourra être résolu qu'en exportant par bateau, ce qui n'est possible que par l'accroissement de la production par expansion de sa culture dans les autres régions du pays (Zou, collines, Ouémé, plateau, Mono et Couffo) où elle est potentiellement possible et en améliorant significativement sa productivité. De plus, l'État peut revoir aussi les frais de manutention et de transit qui sont encore très élevés en les réduisant au quart de leurs valeurs actuelles respectives.

Etant donné que les producteurs de jus en sont les exportateurs, ces ratios indiquent aussi que les différences des prix de vente au niveau national et au niveau des pays importateurs sont faibles et les frais de manutention et de transport faibles. De cette manière, ils perçoivent mieux les parts de richesses ainsi générées que les producteurs d'ananas frais.

Tableau 4. Coefficients de protection nominale du produit (CPNP) de l'exportation de l'ananas frais et du jus vers les pays de l'hinterland, le Nigeria, le Sénégal et l'Europe.

\begin{tabular}{|c|c|c|c|c|c|c|c|}
\hline \multirow[t]{2}{*}{ Paramètres } & \multirow[t]{2}{*}{ Modalités } & \multicolumn{3}{|c|}{ Ananas frais } & \multicolumn{3}{|c|}{ Jus d'ananas } \\
\hline & & $\begin{array}{c}\text { Pays de } \\
\text { l'hinterland }\end{array}$ & Nigeria & Europe & $\begin{array}{c}\text { Pays de } \\
\text { l'hinterland }\end{array}$ & Nigeria & Sénégal \\
\hline \multirow[t]{3}{*}{ Genre } & Homme & $0,065(0,022)$ & $\begin{array}{r}0,092 \\
(0,031)\end{array}$ & $\begin{array}{r}0,037 \\
(0,012)\end{array}$ & $0,686(0,115)$ & $\begin{array}{r}0,656 \\
(0,110)\end{array}$ & $\begin{array}{r}0,514 \\
(0,086)\end{array}$ \\
\hline & Femme & $0,075(0,036)$ & $\begin{array}{r}0,106 \\
(0,051)\end{array}$ & $\begin{array}{r}0,043 \\
(0,021)\end{array}$ & $0,651(0,130)$ & $\begin{array}{r}0,623 \\
(0,124)\end{array}$ & $\begin{array}{r}0,488 \\
(0,098) \\
\end{array}$ \\
\hline & F de Fischer & 2,625 & 2,626 & 2,631 & 0,827 & 0,827 & 0,827 \\
\hline \multirow[t]{6}{*}{$\begin{array}{c}\text { Niveau } \\
\text { d'instruction }\end{array}$} & Aucun & $0,066(0,018)$ & $\begin{array}{r}0,093 \\
(0,025)\end{array}$ & $\begin{array}{r}0,037 \\
(0,010)\end{array}$ & & & \\
\hline & Primaire & $0,067(0,034)$ & $\begin{array}{r}0,095 \\
(0,047)\end{array}$ & $\begin{array}{r}0,038 \\
(0,019)\end{array}$ & $0,713(0,099)$ & $\begin{array}{r}0,683 \\
(0,094)\end{array}$ & $\begin{array}{r}0,535 \\
(0,074)\end{array}$ \\
\hline & $\begin{array}{l}\text { Secondaire } 1^{\text {er }} \\
\text { cycle }\end{array}$ & $0,066(0,018)$ & $\begin{array}{r}0,093 \\
(0,025)\end{array}$ & $\begin{array}{r}0,037 \\
(0,010)\end{array}$ & $0,610(0,117)$ & $\begin{array}{r}0,584 \\
(0,112)\end{array}$ & $\begin{array}{r}0,457 \\
(0,088)\end{array}$ \\
\hline & $\begin{array}{l}\text { Secondaire } 2^{\text {nd }} \\
\text { cycle }\end{array}$ & $0,060(0,024)$ & $\begin{array}{r}0,084 \\
(0,034)\end{array}$ & $\begin{array}{r}0,034 \\
(0,014)\end{array}$ & $0,681(0,170)$ & $\begin{array}{r}0,651 \\
(0,163)\end{array}$ & $\begin{array}{r}0,510 \\
(0,128)\end{array}$ \\
\hline & Supérieur & $0,062(0,113)$ & $\begin{array}{r}0,088 \\
(0,016)\end{array}$ & $\begin{array}{r}0,036 \\
(0,006)\end{array}$ & $\begin{array}{r}0,6485 \\
(0,0930)\end{array}$ & $\begin{array}{r}0,621 \\
(0,089)\end{array}$ & $\begin{array}{r}0,486 \\
(0,070)\end{array}$ \\
\hline & F de Fischer & 0,518 & 0,516 & 0,517 & 0,518 & 0,518 & 0,518 \\
\hline \multirow[t]{3}{*}{ Variétés } & Cayenne lisse & $0,076(0,024)$ & $\begin{array}{r}0,107 \\
(0,034)\end{array}$ & $\begin{array}{r}0,043 \\
(0,014)\end{array}$ & & & \\
\hline & Pain de sucre & $0,064(0,022)$ & $\begin{array}{r}0,091 \\
(0,032)\end{array}$ & $\begin{array}{r}0,036 \\
(0,013)\end{array}$ & & & \\
\hline & F de Fischer & $12,541 * * *$ & $12,52 * * *$ & $12,571 * * *$ & & & \\
\hline \multirow[t]{3}{*}{$\begin{array}{l}\text { Technologie } \\
\text { utilisée }\end{array}$} & Non motorisée & & & & $0,635(0,085)$ & $\begin{array}{r}0,607 \\
(0,081)\end{array}$ & $\begin{array}{r}0,476 \\
(0,064)\end{array}$ \\
\hline & Motorisée & & & & $0,684(0,143)$ & $\begin{array}{r}0,654 \\
(0,137)\end{array}$ & $\begin{array}{r}0,513 \\
(0,107)\end{array}$ \\
\hline & F de Fischer & & & & 1,691 & 1,691 & 1,691 \\
\hline Ensemble & Ensemble & $0,066(0,023)$ & $\begin{array}{r}0,093(0,3 \\
23)\end{array}$ & $\begin{array}{r}0,037 \\
(0,013)\end{array}$ & $0,664(0,124)$ & $\begin{array}{r}0,635 \\
(0,119)\end{array}$ & $\begin{array}{r}0,498 \\
(0,093)\end{array}$ \\
\hline
\end{tabular}

Source : Données de l'enquête, 2013.

( ) Ecart-type ; *** significatif à $1 \%$. 


\subsection{Coefficients de protection effective (CPE) de l'exportation de l'ananas et du jus}

Le coefficient de protection effective (CPE), qui est le rapport de la différence des chiffres d'affaires et des coûts des facteurs échangeables au prix du marché sur cette même différence au prix de référence, mesure les effets des distorsions des marchés du produit et de ceux des intrants échangeables simultanément. Ils indiquent, de ce fait, une distribution effective des revenus en défaveur des producteurs ou une valeur ajoutée moins perçue par ces derniers que ce qu'ils devraient percevoir s'ils étaient liés aux prix des marchés extérieurs. Ils sont alors taxés et ce sont les commerçants et les autres services de commercialisation qui profitent des coûts d'opportunité de l'exportation vers ces différents pays ou régions. Pour l'ananas frais, ces coefficients de protection effective sont statistiquement différents suivant le genre (au seuil de 5\%) et suivant les variétés cultivées (au seuil de 1\%). Ainsi, par exemple, par l'exportation de l'ananas vers l'Europe, les valeurs ajoutées sur les facteurs échangeables des producteurs ne représentent effectivement que $6,27 \%$ de la valeur ajoutée économique créée sur ces facteurs échangeables. Les frais élevés des frets, de manutention, de transit à l'exportation sapent les valeurs ajoutées créées par les facteurs de production qui sont les engrais et les pesticides et défavorisent les producteurs, ce qui ne leur permet pas de mieux profiter des avantages procurés par ces échanges. Ces frais d'exportation variables suivant le volume des exportations doivent être réduits pour accroître les prix aux producteurs qui sont les acteurs qui subissent durement cette distorsion.

Par rapport au jus, ces CPE varient aussi suivant les catégories de producteurs de jus et suivant les pays importateurs. Ils expriment que les producteurs de jus perçoivent respectivement $63,58 \% ; 57,1 \%$ et $49,95 \%$ des valeurs ajoutées sur les facteurs échangeables de jus en l'exportant vers les pays de l'hinterland, le Nigeria et le Sénégal respectivement. Ces coefficients étant inférieurs à l'unité, ils expriment une distribution en défaveur des producteurs-exportateurs de jus et montrent que l'exportation du jus est taxée. Ces coefficients moyens sont statistiquement différents au seuil de $10 \%$ pour l'hinterland et le Nigeria suivant les technologies utilisées et ils ont pour moyennes respectives 0,$6358 ; 0,5710$ et 0,4995 pour les pays de l'hinterland, le Nigeria et le Sénégal (Tableau 5).

Cependant, on constate que ces CPE sont plus élevés que ceux de l'exportation de l'ananas frais. Ainsi, il y a une distribution des revenus plus en faveur des producteurs de jus que ceux de l'ananas frais. Les producteurs de jus étant les exportateurs, les valeurs ajoutées, sur intrants échangeables, sont plus concentrées dans leurs mains. De plus, les charges d'exportation du jus sont moins élevées et pèsent moins sur ces valeurs ajoutées, ce qui fait que les valeurs de ces CPNP et les CPE soient proches. De cette façon, on 
pourrait conclure que ces CPE sont dus à la faible différence des prix entre le pays producteur de jus et les pays importateurs. Cette faible différence de prix est essentiellement le reflet de la forte concurrence des boissons sucrées dans la sous-région. La recherche d'autres marchés de vente de jus plus rémunérateurs s'impose. Mais ceci ne sera possible que grâce à une meilleure organisation des producteurs et une harmonisation des techniques et procédés de production du jus (ABC/SNV, 2016).

Tableau 5. Coefficients de protection effective (CPE) moyens suivant les pays importateurs de l'ananas et de son jus et quelques paramètres liés aux producteurs.

\begin{tabular}{|c|c|c|c|c|c|c|c|}
\hline \multirow[t]{2}{*}{ Paramètres } & \multirow[t]{2}{*}{ Modalités } & \multicolumn{3}{|c|}{ Ananas frais } & \multicolumn{3}{|c|}{ Jus d'ananas } \\
\hline & & $\begin{array}{r}\text { Pays de } \\
\text { l'hinterland }\end{array}$ & Nigeria & Europe & $\begin{array}{r}\text { Pays de } \\
\text { l'hinterland }\end{array}$ & Nigeria & $\begin{array}{r}\text { Sénéga } \\
1\end{array}$ \\
\hline \multirow[t]{3}{*}{ Genre } & Homme & $\begin{array}{r}0,060 \\
(0,024)\end{array}$ & $\begin{array}{r}0,083 \\
(0,033)\end{array}$ & $\begin{array}{r}0,062 \\
(0,025)\end{array}$ & $\begin{array}{r}0,665 \\
(0,182)\end{array}$ & $\begin{array}{r}0,581 \\
(0,147)\end{array}$ & $\begin{array}{r}0,456 \\
(0,103)\end{array}$ \\
\hline & Femme & $\begin{array}{r}0,073 \\
(0,039)\end{array}$ & $\begin{array}{r}0,101 \\
(0,054)\end{array}$ & $\begin{array}{r}0,075 \\
(0,040)\end{array}$ & $\begin{array}{r}0,618 \\
(0,280)\end{array}$ & $\begin{array}{r}0,565 \\
(0,302)\end{array}$ & $\begin{array}{r}0,526 \\
(0,586)\end{array}$ \\
\hline & $\mathrm{F}$ de Fischer & $4,342 * *$ & $4,338 * *$ & $4,16^{* *}$ & 0,377 & 0,041 & 0,234 \\
\hline \multirow{6}{*}{$\begin{array}{c}\text { Niveau } \\
\text { d'instructio } \\
\mathbf{n}\end{array}$} & Aucun & $\begin{array}{r}0,061 \\
(0,020)\end{array}$ & $\begin{array}{r}0,084 \\
(0,027)\end{array}$ & $\begin{array}{r}0,063 \\
(0,020)\end{array}$ & & & \\
\hline & Primaire & $\begin{array}{r}0,062 \\
(0,036)\end{array}$ & $\begin{array}{r}0,086 \\
(0,049)\end{array}$ & $\begin{array}{r}0,064 \\
(0,037)\end{array}$ & $\begin{array}{r}0,726 \\
(0,116)\end{array}$ & $\begin{array}{r}0,649 \\
(0,103)\end{array}$ & $\begin{array}{l}0,521 \\
(0,08)\end{array}$ \\
\hline & $\begin{array}{l}\text { secondaire } 1^{\text {er }} \\
\text { cycle }\end{array}$ & $\begin{array}{r}0,059 \\
(0,019)\end{array}$ & $\begin{array}{r}0,081 \\
(0,026)\end{array}$ & $\begin{array}{r}0,061 \\
(0,019)\end{array}$ & $\begin{array}{r}0,595 \\
(0,124)\end{array}$ & $\begin{array}{r}0,537 \\
(0,107)\end{array}$ & $\begin{array}{r}0,428 \\
(0,078)\end{array}$ \\
\hline & $\begin{array}{l}\text { Secondaire } 2^{\text {nd }} \\
\text { cycle }\end{array}$ & $\begin{array}{r}0,057 \\
(0,025)\end{array}$ & $\begin{array}{r}0,078 \\
(0,035)\end{array}$ & $\begin{array}{r}0,058 \\
(0,026)\end{array}$ & $\begin{array}{r}0,639 \\
(0,278)\end{array}$ & $\begin{array}{r}0,553 \\
(0,238)\end{array}$ & $\begin{array}{r}0,430 \\
(0,175)\end{array}$ \\
\hline & Supérieur & $\begin{array}{r}0,060 \\
(0,013)\end{array}$ & $\begin{array}{r}0,083 \\
(0,017)\end{array}$ & $\begin{array}{r}0,062 \\
(0,013)\end{array}$ & $\begin{array}{r}0,618 \\
(0,275)\end{array}$ & $\begin{array}{r}0,572 \\
(0,317)\end{array}$ & $\begin{array}{r}0,562 \\
(0,683)\end{array}$ \\
\hline & F de Fischer & 0,35 & 0,351 & 0,353 & 0,203 & 0,13 & 0,185 \\
\hline \multirow[t]{3}{*}{ Variétés } & Cayenne lisse & $\begin{array}{r}0,072 \\
(0,025)\end{array}$ & $\begin{array}{r}0,099 \\
(0,034)\end{array}$ & $\begin{array}{r}0,075 \\
(0,026)\end{array}$ & & & \\
\hline & Pain de sucre & $\begin{array}{r}0,059 \\
(0,024)\end{array}$ & $\begin{array}{r}0,081 \\
(0,033)\end{array}$ & $\begin{array}{r}0,061 \\
(0,025)\end{array}$ & & & \\
\hline & F de Fischer & $14,485 * * *$ & $\begin{array}{r}14,46 * * \\
*\end{array}$ & $14,299 * * *$ & & & \\
\hline \multirow[t]{3}{*}{$\begin{array}{l}\text { Technologi } \\
\text { e utilisée }\end{array}$} & $\begin{array}{l}\text { Non } \\
\text { motorisée }\end{array}$ & & & & $\begin{array}{r}0,558 \\
(0,217)\end{array}$ & $\begin{array}{r}0,494 \\
(0,193)\end{array}$ & $\begin{array}{r}0,393 \\
(0,147)\end{array}$ \\
\hline & Motorisée & & & & $\begin{array}{r}0,688 \\
(0,255)\end{array}$ & $\begin{array}{r}0,622 \\
(0,278)\end{array}$ & $\begin{array}{r}0,570 \\
(0,582)\end{array}$ \\
\hline & $\mathrm{F}$ de Fischer & & & & $3,149 *$ & $2,88 *$ & 1,585 \\
\hline Ensemble & & $\begin{array}{r}0,061 \\
(0,025)\end{array}$ & $\begin{array}{r}0,083 \\
(0,034)\end{array}$ & $\begin{array}{r}0,063 \\
(0,026)\end{array}$ & $\begin{array}{r}0,636 \\
(0,246)\end{array}$ & $\begin{array}{r}0,571 \\
(0,253)\end{array}$ & $\begin{array}{r}0,500 \\
(0,465)\end{array}$ \\
\hline
\end{tabular}

Source : Données de l'enquête, 2015.

( ) Ecart-type ; ${ }^{* * *} ; * *$ et * respectivement significatifs à $1 \%, 5 \%$ et $10 \%$.

\section{CONCLUSION}

L'exportation de l'ananas frais et du jus est rentable quelle que soit la zone d'exportation. L'ananas frais exporté vers les pays de l'hinterland est plus rentable que son exportation vers l'Europe. Celle-ci coûte 5,73 fois en ressources locales et elle est découragée par les coûts élevés du fret et des charges de manutention. L'extension de la zone de production et le 
renforcement des capacités des producteurs par l'encadrement permettront d'accroître les volumes exportés et de réduire ces charges. Par contre, l'exportation du jus vers les pays de l'hinterland est moins rentable que celle de l'ananas. Elle est fortement concurrencée par d'autres boissons sucrées produites dans la sous-région. L'accroissement du pourcentage d'ananas transformé en jus, l'harmonisation des procédés de production du jus, l'adoption des équipements appropriés à fort taux d'extraction de jus et l'appui de la recherche pour avoir les variétés plus juteuses permettront d'avoir des volumes de jus nécessaires pour la conquête d'autres marchés africains, asiatiques et européens.

\section{Remerciements :}

Nos profondes gratitudes aux Fonds Compétitifs de l'Université d'Abomey-Calavi pour avoir financé entièrement cette recherche.

\section{References:}

1. ABC/SNV (2016). Etude de la qualité du jus d'ananas béninois dans le contexte du marché régional: Cas des pays de l'hinterland (Burkina Faso, Niger) et de Nigeria et Sénégal. Rapport d'étude 68p.

2. Adégbola, P. Y. \& Arouna, A. (2008). Projet de renforcement de la capacité de l'industrie de la coopérative de transformation de l'ananas Béninois et de production du jus biologique. 56p.

3. Arinloyé, A. A. D. D. (2013). Governance, marketing and innovations in Beninese pineapple supply chains: A survey of smallholder farmers in south Benin. Thesis submitted in fulfilment of the requirements for the degree of doctor at Wageningen University, 2013, 194p.

4. Arouna, A. \& Afomassè, D. (2005). Analyse de la compétitivité de la filière ananas au Bénin. Rapport définitif. INRAB/PAPA. 27p.

5. Banque Mondiale (2008). Rapport sur le développement 2008: Agriculture pour le développement. Washington, DC.

6. Banque Mondiale (2012). La défragmentation de l'Afrique : Approfondissement de l'intégration du commerce régional des biens et services. Washington DC, 196p.

7. Biaou, F.C., Yabi, B. D., Mama, J.V., Biaou, G., Agbossou, E., \& Hounhouigan, D. J. (2016). Cartography of pineapple chains values in Benin: A miss exploited opportunity for regional integration; in AJEES vol $10 n^{\circ} 3$ pp 1-11.

8. Bojnec, S. (2003). "Three concepts of competitiveness measures for livestock production in Central and Eastern Europe", Agriculturae Conspectus Scientificus, vol. 68, no 3, pp. 209-220. 
9. Fabre, P. (1994). « Note méthodologique générale pour l'analyse des de filière » : Division de m'Analyse des Politiques, FAO 35 pp 3480.

10. Gorton, M., Davidova, S., \& Ratinger, T. (2000). "The competitiveness of agriculture in Bulgaria and the Czech Republic vis-à-vis the European Union", Comparative Economic Studies, vol. 42, no 1 , pp. 59-86.

11. Gorton, M. \& Davidova, S. (2001). "The international competitiveness of CEEC agriculture", World Economy, vol. 24, no 2, pp. 185-200.

12. Harris, B. (1982). The marketing of foodgrain in the West African Soudano Sahelian States. ICISAT Indian january.

13. INSAE (2013). Recensement Général de la Population et de l'Habitat $4^{\text {ème }}$ édition Rapport provisoire.

14. Latruffe, L. (2010). « Compétitivité, productivité et efficacité dans les secteurs agricole et agroalimentaire », Éditions OCDE. 69p.

15. Mulder, N., Vialou, A., David, B., Rodriguez, M. \& Castilho, M. (2004). La compétitivité de l'agriculture et des industries agroalimentaires dans le Mercosur et l'Union européenne dans une perspective de libéralisation commerciale. Working Paper/Document de travail no 2004-19, Centre d'études prospectives et d'informations internationales (CEPII), Paris, France.

16. Obi, A., Pote, P., \& Chianu, J. (2011). Market access: Components, interactions and implications in smallholder Agriculture in the Former Homeland Area of South Africa. Innovations as key to the green revolution in Africa, 2011. PP 1161-1167.

17. Pearson, S.R. \& Monke, E. (1989). The Policy Analysis Matrix for Agricultural Development. Cornell University Press Ithaca NY, 279p.

18. Stiglitz, J. E. \& Greenwald, B.C. (1989). Financial market imperfections and productivity growth "working paper series 206, research institute of industrial economics.

19. Timmer, C.P., Falcon, W.C. \& Pearson, S.R. (1986). Analyse de la politique alimentaire ; Economica, Paris 364p.

20. Timmer, C.P. (1986). Getting prices right:-the scope - and limits of agricultural price politicy. Cornell University Press; Ichaca and London 160p. 